IMSc $/ 2000 / 02 / 03$

TIFR/TH/00-08

\title{
HORIZON STATES FOR AdS BLACK HOLES
}

\author{
T. R. Govindarajan ${ }^{a}$ 円, V.Suneeta ${ }^{a}{ }^{2}$ and S. Vaidya ${ }^{b}$ \\ ${ }^{a}$ Institute of Mathematical Sciences, \\ Chennai, 600 113, India. \\ ${ }^{b}$ Tata Institute of Fundamental Research, \\ Colaba, Mumbai, 400 005, India.
}

\begin{abstract}
We study the time-independent modes of a massless scalar field in various black hole backgrounds, and show that for these black holes, the time-independent mode is localized at the horizon. A similar analysis is done for time-independent, equilibrium modes of the five-dimensional plane AdS black hole. A self-adjointness analysis of this problem reveals that in addition to the modes corresponding to the usual glueball states, there is a discrete infinity of other equilibrium modes with imaginary mass for the glueball. We suggest these modes may be related to a Savvidy-Nielsen-Olesen-like vacuum instability in QCD.
\end{abstract}

\section{Introduction}

A study of various kinds of matter fields propagating in black hole backgrounds yields information about diverse classical and quantum aspects of black hole physics. Detailed analysis of modes of the scalar, spinor and gauge fields in black hole backgrounds can be found for example, in [1]. In particular, for scalar fields, the energies of these modes are given by the square root of the eigenvalues of the spatial part of the Klein-Gordon operator in that background. For static spacetimes with null singularities, it has been argued [2, 3] that the spatial part of the Klein-Gordon operator is essentially self-adjoint. Further, since it is positive and symmetric, one can choose a positive self-adjoint extension such that the eigenvalues are all positive and hence the energies real. However, as we show in this paper, in the case of many black hole spacetimes, near the null singularity at the horizon, the zero (time-independent) mode of the scalar field has to be handled separately. In particular, the boundary conditions imposed on the

\footnotetext{
${ }^{1}$ trg@imsc.ernet.in

${ }^{2}$ suneeta@imsc.ernet.in

${ }^{3}$ sachin@theory.tifr.res.in
} 
zero mode both at the horizon and at infinity are different from those on the other modes with real energies. In fact, we will show that there are an infinite number of boundary conditions, labeled by a $U(1)$ parameter, that lead to one zero mode solution. This solution could be thought of as a 'horizon state' as it is localized at the horizon.

An application of this analysis to the time-independent solutions of the infinite mass limit of the AdS-Schwarzschild black hole [4] leads to results that could be interesting in light of the AdS/CFT correspondence. As proposed by Witten [5], the AdS/CFT duality relating supergravity on anti-de Sitter space to a supersymmetric Yang-Mills theory on the boundary can be extended to non-supersymmetric $Q C D$. The AdS background is replaced by an AdS-Schwarzschild black hole background. It has been shown that gravity on this background gives many of the features of strong coupling limit of $Q C D$, like the area law behavior of Wilson loops, confinement, and the glueball mass spectrum with a mass gap [5 8 .

The glueball mass spectrum is reproduced by certain time-independent and normalizable modes obtained by solving the dilaton wave equation in the black hole geometry. These modes were numerically computed first in [6, [7]. These modes are "equilibrium modes" for the black hole, i.e. the current vanishes at the horizon, which has been recognised in [8] as the correct boundary condition to be used at the horizon.

It has been argued that "non-equilibrium" modes of the same black hole (with ingoing boundary conditions at the horizon) give the time scale of approach to thermal equilibrium of the boundary Yang-Mills theory. These modes, i.e. the quasi-normal modes of the black hole, have been computed recently [9, 10].

In this paper, we study the scalar wave equation in the AdS-Schwarzschild background, and show, that written as a Hamiltonian problem, it is not self-adjoint. Self-adjointness and completeness requires inclusion of modes ignored in 6 8]. These modes are also equilibrium modes of the black hole but are irregular at the horizon 1 . They are also tachyonic. We suggest that these modes are dual in the AdS/CFT sense to modes in $Q C D_{3}$ signaling the onset of a Savvidy-Nielsen-Olesen-like instability of the vacuum [11 14.

The organization of the paper is as follows. In section 2, we briefly describe two kinds of modes that are commonly discussed in related literature, namely the normalizable equilibrium modes, and the non-normalizable quasi-normal modes, to emphasize the differences between them. We also

\footnotetext{
${ }^{4}$ In $[7,8]$, the existence of irregular modes is mentioned. However, they are not considered.
} 
show that for a massless scalar field propagating in Schwarzschild or ReissnerNordstrom black hole background, the Klein-Gordon operator is self-adjoint. In section 3, we focus on the zero energy mode of the scalar field in these backgrounds, and in the background of the (1+1)-d black hole [15] as well as the BTZ black hole [16]. The equation obeyed by the zero mode has unusual properties, which we analyze in section 4 . In particular, we show that this state localized at the horizon. In section 5, we apply the results of section 4 to study the zero mode of the massless scalar field in the background of the infinite mass limit of the AdS-Schwarzschild black hole, and argue that the "horizon states" are necessary for completeness. In section 7 , we speculate on the interpretation of these irregular modes in the boundary theory, and suggest that they may be related to a Savvidy-Nielsen-Olesen-like instability.

\section{Modes of the scalar field in black hole back- ground}

As mentioned before, the energies of normalizable modes of a scalar field in the exterior of a black hole spacetime (i.e. in the region from the outer horizon to infinity) have real energies.

This can be verified for the Schwarzschild or Reissner-Nordstrom black hole in the exterior. There are no normalizable mode solutions with complex (or pure imaginary) energies. However, this is not true in a region of the black-hole spacetime near a timelike singularity. For the Reissner-Nordstrom spacetime, in the region between the timelike singularity and the inner horizon, the spatial part of the Klein-Gordon operator is not self-adjoint, as also observed by [17], but can be made self-adjoint by a suitable choice of boundary conditions. There exist boundary conditions for which there is a negative eigenvalue for this operator, leading to a mode solution with imaginary energy. However, this solution is not extendible to the physical region of interest between the outer horizon and infinity.

Other modes of importance in the context of black holes are the quasinormal modes (see for example, [18]). For the case of asymptotically flat black holes, these are defined to be purely ingoing near the horizon and outgoing at infinity. These are not normalizable, but are of interest as their energies are the characteristic frequencies associated with the perturbation of the black hole. These are in general, complex, and decay with time. In the Schwarzschild and Reissner-Nordstrom cases, there are an infinite number of such modes (see [18] for references) which include purely imaginary modes [19. 
Recently, quasi-normal modes for the AdS-Schwarzschild black hole have also been studied [10]. These are different from the quasi-normal modes for asymptotically flat black holes, in that they are not outgoing at infinity, but vanish. This is due to the fact that the AdS potential diverges at infinity. Numerical results of [10] suggest that these modes are complex. However, they are still non-normalizable due to their behavior at the horizon.

An analysis of the spatial part of the Klein-Gordon operator for the AdS-Schwarzschild black hole shows that as expected in [3], the operator is self-adjoint, and all the normalizable modes have real energies. The AdSSchwarzschild black hole has a metric

$$
\begin{aligned}
d s^{2} & =-F(r) d \tau^{2}+F^{-1}(r) d r^{2}+r^{2} d \Omega^{2}, \quad \text { where } \\
F(r) & =\left(1+r^{2} / b^{2}-r_{0}^{2} / r^{2}\right) .
\end{aligned}
$$

Here $b$ is the radius of curvature of the anti-de Sitter space and $r_{0}$ is related to the black hole mass,

$$
M=\frac{3 A_{3} r_{0}^{2}}{16 \pi G_{5}}
$$

and $A_{3}$ is the area of a unit 3 -sphere.

Let us look at a massless scalar field in this background geometry. One can in principle consider a complex scalar field with charge $q$ and mass $m$, but for simplicity we shall consider only the massless and uncharged field in the black hole background. The action for such a field $\Phi$ is

$$
\begin{aligned}
S & =-\frac{1}{2} \int \sqrt{|g|} g^{i j}\left(\partial_{i} \Phi\right)\left(\partial_{j} \Phi\right) d^{5} x \\
& =-\frac{1}{2} \int_{r_{+}}^{\infty} d r \int d t \int d \Omega\left[r^{3}\left\{\frac{\dot{\Phi}^{2}}{F}+F \Phi^{\prime 2}+\left(1 / r^{2}\right) \Phi L^{2} \Phi\right\}\right] .
\end{aligned}
$$

The Klein-Gordon equation for the field $\Phi$ can be obtained from above. On making the ansatz

$$
\Phi=\frac{f(r)}{r^{3 / 2}} Y(\text { angles }) \exp -(i \omega t),
$$

the wave functions are defined on the measure $d r / F$. The Klein-Gordon equation can then be written in terms of the tortoise coordinate $r_{*}$, which is defined by $d r_{*}=d r / H$. It takes the form

$$
-\frac{d^{2}}{d r_{*}^{2}} f+V\left(r_{*}\right) f=\omega^{2} f,
$$


with the measure now being $d r_{*}$. The potential is positive, vanishes at the horizon $r_{*}=-\infty$ and diverges at $r=\infty$. This corresponds to a finite $r_{*}$ and therefore the solutions have to vanish there. Multiplying (2.6) by the complex conjugate of $f$ and integrating over the spacetime from the horizon to infinity, it can be seen that there can be no normalizable solutions that correspond to $\omega^{2}$ negative or complex. This is in conformity with the fact that the Klein-Gordon operator is self-adjoint.

\section{Time independent mode in black hole solutions}

The positive energy solutions to the Klein-Gordon equation can be analyzed for most black hole solutions by going to the tortoise coordinate $r_{*}$ mentioned in the previous section. The solutions behave as $f \sim \exp i \omega\left(t \pm r_{*}\right)$ near the horizon and near infinity. The horizon is at $r_{*}=-\infty$, while the infinity of the Schwarzschild radial coordinate is either at $r_{*}=\infty$ or at a finite $r_{*}$, depending on the black hole considered. The solutions are plane wave normalizable, and have infinitely oscillating phases at the horizon.

The near-horizon analysis of black hole solutions reveals, however, that the time independent $(\omega=0)$ mode of the scalar field has to be handled carefully.

The metric for an asymptotically flat, spherically symmetric, static black hole in 4 -D is of the form

$$
d s^{2}=-F(r) d t^{2}+F^{-1}(r) d r^{2}+r^{2} d \Omega^{2} \equiv g_{i j} d x^{i} d x^{j} .
$$

For a Reissner-Nordstrom black hole,

$$
\begin{aligned}
F(r) & =-\frac{\left(r-r_{+}\right)\left(r-r_{-}\right)}{r^{2}} \\
r_{ \pm} & =Q l_{P}+E l_{P} \pm\left(2 Q E l_{P}^{3}+E^{2} l_{P}^{4}\right)^{2} .
\end{aligned}
$$

Here, $l_{P}$ is the Planck length and $E=M-Q / l_{P}$ is the energy above extremality. For a Schwarzschild black hole, $F(r)=(1-2 M / r)$.

Let us look at a massless scalar field in this background geometry. The action for such a field $\phi$ is

$$
S=-\frac{1}{2} \int \sqrt{|g|} g^{i j} \partial_{i} \phi \partial_{j} \phi
$$

If we restrict out attention to spherically symmetric configurations, the action looks like

$$
S=-\frac{1}{2} \int\left[-(\dot{\phi})^{2}+F^{2}(r)\left(\phi^{\prime}\right)^{2}\right] \frac{d r}{F(r)} d t .
$$


This immediately allows us to identify the Lagrangian:

$$
L=\frac{1}{2} \int \frac{d r}{F(r)}\left[(\dot{\phi})^{2}-F(r)^{2}\left(\phi^{\prime}\right)^{2}\right] .
$$

The modes of the scalar field are obtained from the ansatz that the time dependence of $\phi$ is $\phi \sim \exp (i \omega t)$. We are interested in the time-independent solutions, so we take $\omega=0$. Then the Klein-Gordon equation for this case is obtained simply by considering the second term in the Lagrangian, and is

$$
H=-\frac{1}{F} \frac{d}{d r}\left(F \frac{d}{d r}\right) \psi=0,
$$

where wave functions are defined on $L^{2}\left[(0, \infty), r^{2} F d r\right]$. It is more convenient to work with the measure $d r$ rather than $r^{2} F(r) d r$, so we make a unitary transformation from $L^{2}\left[\mathbb{R}^{+}, F(r) d r\right]$ to $L^{2}\left[\mathbb{R}^{+}, d r\right]$ via $U \psi=\sqrt{r^{2} F(r)} \psi=\chi$. In this new basis, $H$ reads:

$$
H=-\frac{d^{2} \chi}{d r^{2}}+\left[\frac{\left(r^{2} F\right)^{\prime \prime}}{2 F}-\left(\frac{\left(r^{2} F\right)^{\prime}}{2 F}\right)^{2}\right] \chi=0 .
$$

On putting the value of $F$ for the black hole in (3.8) and taking the near-horizon limit, we find that both for the non-extremal black holes, (3.8) in the near-horizon limit is

$$
\left(-\frac{d^{2}}{d x^{2}}-\frac{1}{4 x^{2}}\right) \chi=0
$$

where $x=\left(r-r_{+}\right)$is the near-horizon coordinate. $r_{+}$is the horizon. For the extremal Reissner-Nordstrom solution, however, (3.8) reduces near the horizon to

$$
-\frac{d^{2} \chi}{d x^{2}}=0
$$

Another situation where we see a similar equation is the near horizon geometry of the one-dimensional black hole discovered by Witten [15]. The metric for this black hole is of the form

$$
d s^{2}=-\tanh ^{2}(r / R) d t^{2}+d r^{2} .
$$

The action for a scalar field propagating in this background is

$$
S=-1 / 2 \int \sqrt{|g|} g^{i j} \partial_{i} \phi \partial_{j} \phi d r d t
$$


The Lagrangian is

$$
L=1 / 2 \int \tanh (r / R)\left[\frac{\dot{\phi}^{2}}{\tanh ^{2}(r / R)}-\phi^{\prime 2}\right] d r .
$$

The Klein-Gordon equation for the zero mode can be calculated from the 2nd term, the functions being defined on $L^{2}[(0, \infty), \tanh (r / R) d r]$ :

$$
-\frac{1}{\tanh (r / R)} \frac{d}{d r}\left[\tanh (r / R) \frac{d}{d r}\right] \psi=0 .
$$

Again, we can make a unitary transformation from $L^{2}\left[\mathbb{R}^{+}, \tanh (r / R) d r\right]$ to $L^{2}\left[\mathbb{R}^{+}, d r\right]$ via $U \psi=\sqrt{\tanh (r / R)} \psi=\chi$, the equation now is

$$
-\frac{d^{2} \chi}{d r^{2}}+\frac{1}{R^{2}}\left[\frac{-1 / 4}{\tanh ^{2}(r / R)}+\frac{3}{4} \tanh ^{2}(r / R)-\frac{1}{2}\right] \chi=0 .
$$

For small $r$, the equation is approximately

$$
-\frac{d^{2} \chi}{d r^{2}}-\left[\frac{1}{4 r^{2}}+\frac{1}{2 R^{2}}\right] \chi=0 .
$$

Another black hole that exhibits the same behavior is the BTZ black hole in $(2+1)-D$ gravity [16]. For simplicity, we take $J=0$. It has a metric given by

$$
d s^{2}=-N^{2} d t^{2}+1 / N^{2} d r^{2}+r^{2} d \phi^{2},
$$

where $N^{2}=\left(r^{2} / l^{2}-M\right),-1 / l^{2}$ is the curvature of AdS space and $M$ is the black hole mass. Here, again, the near horizon Klein- Gordon equation is

$$
-\frac{d^{2} \psi}{d x^{2}}-\frac{\psi}{4 x^{2}}=0
$$

where $(r-l \sqrt{M})=x$ is the near-horizon coordinate. In the case of the Schwarzschild, non-extremal and Reissner-Nordstrom equations, the nextorder correction is of order $1 /\left(r-r_{+}\right)$.

Thus, in all these cases barring the extremal RN black hole, (3.18) is the near-horizon equation for the zero-mode solution. The solutions, both to (3.18) and the extremal case are discussed in the next section. The eigenvalues of the Hamiltonian which is just the 1.h.s of (3.18) and of the operator which is the l.h.s of (3.10) are obtained. The solutions of interest are the zero eigenvalue solutions for that Hamiltonian problem. We will see that the self-adjointness analysis of the Hamiltonian $H$, i.e the l.h.s operator in (3.18) will help us find these solutions. 


\section{Self-Adjointness of the operator $H$}

As is well-known (see for example 20, 21]), discussion of self-adjointness (or "hermiticity") for an unbounded operator $\mathcal{O}$ first requires us to define the domain $D(\mathcal{O})$ of $\mathcal{O}$. We will only be interested in operators that are defined on domains that are dense in the Hilbert space. This allows us to define $\mathcal{O}^{*}$, the adjoint of $\mathcal{O}$, and $D\left(\mathcal{O}^{*}\right)$. By definition, $\mathcal{O}$ is self-adjoint if and only if $D(\mathcal{O})=D\left(\mathcal{O}^{*}\right)$. A better way of saying this is by looking at "deficiency indices", which are defined as follows. Let $\mathcal{K}_{ \pm}=\operatorname{Ker}\left(i \pm \mathcal{O}^{*}\right)$, where $\operatorname{Ker}(X)$ is the kernel of the operator $X$. The integers $n_{ \pm} \equiv \operatorname{dim} \mathcal{K}_{ \pm}$ are the deficiency indices of the operator. If $n_{ \pm}=0$, then $\mathcal{O}$ is essentially self-adjoint. If $n_{+}=n_{-}=n \neq 0$, the $\mathcal{O}$ is not self-adjoint but has selfadjoint extensions. Different self-adjoint extensions of the operator are in one-one correspondence with unitary maps from $\mathcal{K}_{+}$to $\mathcal{K}_{-}$, that is, they are labeled by a $U(n)$ matrix. Finally, if $n_{+} \neq n_{-}$, then $\mathcal{O}$ cannot be made self-adjoint.

The Hamiltonian $H$ is a special case of a more general Hamiltonian studied extensively in the literature. It is defined on a domain $L^{2}\left[\mathbb{R}^{+}, d x\right]$ and is of the form

$$
H_{\alpha}=-\frac{d}{d x^{2}}+\frac{\alpha}{x^{2}}
$$

Classically, the system described by this Hamiltonian is scale invariant ( $\alpha$ is a dimensionless constant). However, the quantum analysis of this operator is much more subtle. As was shown by [22,23], $H_{\alpha}$ is essentially self- adjoint only for $\alpha>3 / 4$. For $\alpha>3 / 4$, the domain of the Hamiltonian is

$$
\mathcal{D}_{0}=\left\{\psi \in \mathcal{L}^{2}(d x), \psi(0)=\psi^{\prime}(0)=0\right\}
$$

For $\alpha \leq 3 / 4$, this operator is not essentially self-adjoint (and therefore cannot play the role of a Hamiltonian) and so has to be "extended" to another operator. For this case, the deficiency indices are $\langle 1,1\rangle$, and so the self-adjoint extensions are labeled by a $U(1)$ parameter $e^{i z}$, which labels the domains $\mathcal{D}_{z}$ of the Hamiltonian $H_{z}$. The set $\mathcal{D}_{z}$ contains all the vectors in $\mathcal{D}_{0}$, and vectors of the form $\psi_{+}+e^{i z} \psi_{-}$, where

$$
\begin{gathered}
\psi_{+}=x^{1 / 2} H_{\nu}^{(1)}\left(x e^{i \pi / 4}\right), \\
\psi_{-}=x^{1 / 2} H_{\nu}^{(2)}\left(x e^{-i \pi / 4}\right),
\end{gathered}
$$

where $\nu=\sqrt{1 / 4+\alpha}$, and $H_{\nu}^{(1,2)}$ 's are the Hankel functions $J_{\nu} \pm i N_{\nu}$. The 
small $x$ behavior of $\psi_{+}+e^{i z} \psi_{-}$is

$$
\begin{aligned}
\psi_{+}+e^{i z} \psi_{-} \sim & \frac{i x^{1 / 2}}{\sin (\pi \nu)}\left[\left(\frac{x}{2}\right)^{\nu} \frac{e^{-3 \pi i \nu / 4}-e^{-i z+3 \pi i \nu / 4}}{\Gamma(1+\nu)}\right. \\
& \left.+\left(\frac{x}{2}\right)^{-\nu} \frac{e^{i z+i \pi \nu / 4}-e^{-i \pi \nu / 4}}{\Gamma(1-\nu)}\right]
\end{aligned}
$$

We can now solve the eigenvalue equation for bound states:

$$
-\psi^{\prime \prime}+\frac{\alpha}{x^{2}} \psi=-E \psi \text {. }
$$

For $\alpha \geq 3 / 4$, there are no bound states. More precisely, there are no normalizable solutions to the Schrödinger equation with negative energy. However, for $-1 / 4 \leq \alpha<3 / 4$ there is exactly one bound state of energy $E_{b}$, where $E_{b}$ is

$$
E_{b}=E(\nu, z)=\left[\frac{\sin (z / 2+3 \pi \nu / 4)}{\sin (z / 2+\pi \nu / 4)}\right]^{1 / \nu},
$$

and the corresponding eigenfunction is

$$
\psi=N\left(\sqrt{E_{b}} x\right)^{1 / 2}\left[J_{\nu}\left(i \sqrt{E_{b}} x\right)-e^{i \pi \nu} J_{-\nu}\left(i \sqrt{E_{b}} x\right)\right] .
$$

The existence of bound states seems to be in contradiction with scale invariance, since scale invariance implies that there is no length scale in the problem, whereas the existence of the bound state provides a scale. This tension can be resolved by looking at how scaling is implemented in the quantum theory. The scaling operator is

$$
\Lambda=\frac{x p+p x}{2}
$$

where $p=-i d / d x$. It is easily seen that $\Lambda$ is symmetric on the domain $\mathcal{D}$ of $H$, and that for $\alpha>3 / 4, \Lambda$ leaves invariant the domain of the Hamiltonian. For $\alpha \leq 3 / 4$,

$$
\Lambda \psi=x^{3 / 2}\left[\psi_{+}+e^{i z} \psi_{-}\right]^{\prime}
$$

The small $x$ behavior of the function $\Lambda \psi$ is of the form

$$
\begin{aligned}
\Lambda \psi & \simeq \frac{-i \nu x^{1 / 2}}{\sin \pi \nu}\left[\left(\frac{x}{2}\right)^{\nu}\left(2 e^{i \pi / 4}-1\right)\left(\frac{e^{-3 \pi i \nu / 4}-e^{i \tilde{z}+3 \pi i \nu / 4}}{\Gamma(1+\nu)}\right)\right. \\
& \left.+\left(\frac{x}{2}\right)^{-\nu}\left(\frac{e^{i \tilde{z}+i \pi \nu / 4}-e^{-i \pi \nu / 4}}{\Gamma(1-\nu)}\right)\right]+\cdots,
\end{aligned}
$$


where $\tilde{z}=z+\pi / 2$. So $\Lambda \psi$ clearly does not leave the domain of the Hamiltonian invariant. Scale invariance is thus anomalously broken, and this breaking occurs precisely when the Hamiltonian admits non-trivial self-adjoint extensions. This also explains the quantum mechanical emergence of a length scale, namely the bound state energy.

We must remark here that there do exist self-adjoint extensions that preserve scale invariance. For example, if $z=-(\pi \nu / 2)$, then there is no bound state. From the point of view of the domains, the operator $\Lambda$ leaves this domain invariant, implying that scaling can be consistently implemented in the quantum theory.

Now that we know about the subtleties about quantum mechanical evolution in $1 / x^{2}$ potential, we can apply these ideas to our case. The potential near the horizon is like $-1 / 4 x^{2}$ for the problem of interest.

For the $-1 / 4 x^{2}$ potential, there are infinite number of bound states for a given fixed self-adjoint extension $z$. These are given by

$$
\begin{aligned}
\psi_{E_{n}}(x) & =N_{n} \sqrt{x} K_{0}\left(\sqrt{E_{n}} x\right), \quad n \in \mathbb{Z}, \\
E_{n} & =\exp \left[\frac{\pi}{2}(1-8 n) \cot \frac{z}{2}\right], \quad n \in \mathbb{Z} .
\end{aligned}
$$

These are found by solving (4.6) for $\alpha=-1 / 4$ and carefully comparing the behavior of the eigenfunctions with the analog of (4.5) which is

$$
\psi=e^{-i z / 2}\left(x^{1 / 2}+i x^{1 / 2} \ln x\right)+e^{i z / 2}\left(x^{1 / 2}-i x^{1 / 2} \ln x\right) .
$$

Returning to the original problem of finding the zero mode solutions, i.e the solutions to (3.18), we see that demanding self-adjointness of the Hamiltonian gives rise to an infinite number of bound states labeled by an integer $n$. The zero mode solution is obtained from (4.13) in the $n \rightarrow \infty$ limit. In particular, the wave function for the solution to (3.18) near the horizon is

$$
\psi=N_{n} x^{1 / 2}\left(1+\ln \left(\sqrt{E_{n}} x\right)\right) .
$$

where $E_{n}$ is given by (4.13) and $N_{n}$ is an appropriate normalization factor. Then one takes the limit $n \rightarrow \infty$. This leads to a solution that is non-zero only at the horizon, where it peaks, and can be thought of as a 'horizon state'. $E_{n}$ depends on the self-adjointness parameter $z$, which also corresponds to the boundary condition at the horizon. However, in the limit $n \rightarrow \infty$, all boundary conditions lead to the same solution of (3.18). Since (3.18) is the time-independent zero angular momentum mode for the 
scalar field in all the aforementioned black hole backgrounds, the above discussion applies to all those cases. The behavior of the zero mode found by this method matches that of the numerical zero mode solution for the Schwarzschild black hole in Fig.1 (where the horizon is at $r=50$ ) apart from minor errors in the numerical interpolaion.

For the one exception, the extremal Reissner-Nordstrom black hole, the equation (3.10) is easily solved. The corresponding Hamiltonian problem for which the solutions to (3.10) are the zero eigenvalue solutions was considered in the section above. However, it does not lead to the kind of non-trivial boundary conditions for the zero eigenvalue solution as in the other cases. This is because the self-adjointness analysis of that operator yields only one bound state. The bound state vanishes for a particular value of the self-adjointness parameter, as discussed. Therefore, there seems to be no non-trivial zero mode for the extremal black hole.

\section{Time-independent modes in the plane AdS black hole}

Another black hole solution which can be obtained in the infinite mass limit from the AdS-Schwarzschild solution, the plane AdS solution, was discussed in [5]. The metric for the Euclidean AdS-Schwarzschild black hole in the infinite mass limit is of the form

$$
\begin{aligned}
d s^{2} & =F(r) d \tau^{2}+F^{-1}(r) d r^{2}+r^{2} \sum_{i=1}^{3} d x_{i}^{2}, \quad \text { where } \\
F(r) & =\left(r^{2} / b^{2}-b^{2} / r^{2}\right)
\end{aligned}
$$

Let us look at a massless scalar field in this background geometry. One can in principle consider a complex scalar field with charge $q$ and mass $m$, but for simplicity we shall consider only the massless and uncharged field in the black hole background. The action for such a field $\Phi$ is

$$
\begin{aligned}
S & =-\frac{1}{2} \int \sqrt{|g|} g^{i j}\left(\partial_{i} \Phi\right)\left(\partial_{j} \Phi\right) d^{5} x \\
& =-\frac{1}{2} \int_{b}^{\infty} d r \int_{0}^{\beta} d \tau \int_{-\infty}^{\infty} d^{3} x\left[r^{3}\left\{\frac{\dot{\Phi}^{2}}{F}+F(\Phi)^{\prime 2}+1 / r^{2} \sum_{i}\left(\partial_{x_{i}} \Phi\right)^{2}\right\}\right] .
\end{aligned}
$$

This action, where the scalar field is the Type IIB dilaton field, has been discussed in [5 77. Modes for the field which are $\tau$ independent are considered, where $\Phi(r, x)=f(r) \exp (i k . x)$. Then the equation of motion for $f(r)$ 
is

$$
-r^{-1} d / d r\left(r^{3}\left(r^{2}-1 / r^{2}\right)(d f / d r)\right)+k^{2} f=0,
$$

where $b=1$ is taken for simplicity. On demanding normalizability of $f(r)$ w.r.t. the measure $r^{3} d r$ and regularity of the solution at $r=1$, a discrete negative spectrum for $k^{2}$ was obtained. It was identified with the glueball spectrum in the boundary theory.

We show below that one can consider (5.4) as an eigenvalue problem for $k^{2}$ and examine the operator in this equation for self-adjointness. As is well known, a self-adjoint operator has only real eigenvalues, and any wave function in the domain of the operator can be written in terms of its eigenfunctions. We therefore wish to find the complete set of $k$ modes such that any function of compact support in the domain can be expanded in terms of the mode functions. It is seen that the operator is not selfadjoint, but can be extended to a self-adjoint operator. However, more general boundary conditions are required at $r=1$. Then, the spectrum of $k$ is also enlarged to include a discrete infinity of positive $k^{2}$ states, and some negative $k^{2}$ states as well.

The operator of interest is

$$
T=-r^{-1} d / d r\left[r^{3}\left(r^{2}-1 / r^{2}\right) d / d r\right],
$$

where wave functions are defined on a measure $r^{3} d r$.

We can therefore check the operator $T$ for self-adjointness. We first check if it is symmetric, i.e. if $(\psi, T \phi)=\left(T^{*} \psi, \phi\right)$, where $\phi \in D(T)$, and $\psi \in D\left(T^{*}\right)$.

If the operator $T$ is symmetric, it is self-adjoint if $\left(T^{*} \pm i\right) \psi=0$ has no solutions $\psi$ in $D\left(T^{*}\right)$.

But with this measure, we see that the operator is not even symmetric. We therefore consider the measure $r d r$ which from the action (5.4) is the natural measure to consider if one is interested in looking for the eigenvalue problem for the operator (5.5). However, this measure is not enough to guarantee finiteness of the second term in (5.4). Therefore, we take the domain of functions $D(T)$ to consist of $C_{\infty}$, square integrable functions with respect to the measure $r d r$ which fall off at least as $1 / r^{3}$ (or faster than that) that are of compact support. (Actually, it is enough if they fall of as $1 / r^{2+\delta}$ where $\delta>0$. For convenience, we take $\delta=1$, and it does not affect any of the analysis.) 
The self-adjointness question is easier to address after a change in coordinates, following [7]. On making the transformations

$$
\begin{aligned}
r^{2} & =\cosh x, \\
A(x) & =\sqrt{\sinh (2 x)} f(x),
\end{aligned}
$$

the measure becomes $d x / \cosh x$, and (5.4) becomes

$$
\begin{array}{r}
-4 \cosh x d^{2} / d x^{2} A(x)+4 \cosh x A(x)-4 \cosh x A(x) / \sinh (2 x)^{2} \\
=-k^{2} A(x)
\end{array}
$$

In these coordinates, the horizon is at $x=0$. Here, one can define the domain of interest $D(T)$ to consist of $C_{\infty}$, square integrable functions $A(x)$ with respect to the measure $d x / \cosh x$ and which fall off asymptotically at least as $A(x) \sim \exp (-3 x / 2)$. Also, they are of compact support, so $A(x=0)=A^{\prime}(x=0)=0$. Then it can be shown that the operator on the l.h.s of (5.8) is symmetric, however, the domain of the adjoint $T^{*}$ is now any normalizable function. Thus, $D(T) \neq D\left(T^{*}\right)$. The operator is not selfadjoint. Also, $\left(T^{*}+i\right) \psi=0$ and $\left(T^{*}-i\right) \psi=0$ each have one normalizable solution, as can be verified numerically (see Fig.2). If for each eigenvalue $\pm i$, there is exactly one normalizable solution, then the deficiency indices of this operator are $(1,1)$ and it is possible to find self-adjoint extensions for it. Therefore, one can look for the self-adjoint extension of this operator. Since a self-adjoint extension involves only a change of boundary condition at $x=0$, we deal with the near-horizon form of (5.8) for simplicity.

On using the near-horizon ( $x$ small) approximation, (5.8) becomes

$$
-\left(d^{2} / d x^{2}\right) A(x)-\frac{A(x)}{4 x^{2}}=-\frac{\left(k^{2}+1\right) A(x)}{4} .
$$

This looks like a Hamiltonian problem for a potential $-\frac{1}{4 x^{2}}$ (which was discussed extensively in the previous section) with the eigenvalue $-\left(k^{2}+\right.$ 1) $/ 4$.

The results of the previous section can be applied to the case of (5.9) to find the additional states that arise due to the changed boundary condition. They are given by (4.13) with $E_{n}=\left(k^{2}+1\right) / 4$. Thus, there are eigenvalues $k^{2}$ for each $n$, and $n$ is any integer. The eigenvalues also depend on the selfadjoint parameter $z$. There are positive $k^{2}$ eigenvalues. There is a possibility of finding some values of $k^{2}$ with $k^{2}$ negative too, for which $k^{2}<1$.

What has been done above is a near-horizon analysis of (5.8). It is not clear if all of these states are solutions to the complete equation (5.8). However, numerically, there seem to exist normalizable solutions to the complete 
equation for any positive $k^{2}$, provided one also accepts the non-regular solutions that have not been considered by [5 7]. These are seen to be irregular only at the horizon, exactly like the solution in Fig.2. Imposing a particular boundary condition at the horizon demanded by self-adjointness picks out a discrete infinity of normalizable positive $k^{2}$ states as above.

A feature of these modes that is immediately noticeable is that they are irregular at the black hole horizon. However, from considerations of selfadjointness, they are necessary for expressing any arbitrary, regular field configuration in the bulk in terms of a complete set of mode solutions. In fact, the difference of any two of these irregular solutions is regular. This is because the irregular solutions are irregular only at the horizon, where they behave as $f_{k}(r) \sim \ln (k(r-1))$ where $r=1$ is the horizon. Taking the difference of two solutions $f_{k 1}(r)$ and $f_{k 2}(r)$, we see that the resultant solution is regular at the horizon. Therefore, any arbitrary regular field configuration in the bulk can be constructed with regular mode solutions and an even number of irregular mode solutions.

It may seem that the irregular solutions can be gotten rid of by shifting the domain of interest a small distance $\epsilon$ away from the horizon, where $\epsilon>0$ and repeating the self-adjointness analysis for this new domain. However, letting $\epsilon \rightarrow 0$, the irregular solutions reappear. Further, the one parameter ambiguity in boundary conditions is not resolved. Letting $\epsilon \rightarrow 0$ does not pick any particular boundary condition at the horizon [22].

\section{Discussion}

We find that on examining scalar field theory in the background of the infinite mass limit of the AdS-Schwarzschild black hole, there are more timeindependent, equilibrium modes than previously obtained [6, 6]. These are however positive $k^{2}$ modes. There is a parameter labeling the boundary conditions at the horizon (for the self-adjoint extension) on which these modes depend.

We analyzed the time-independent, $L=0$ solutions of the $(3+1)-d$ Schwarzschild and Reissner-Nordstrom black holes, the $(1+1)-d$ dilatonic black hole and the BTZ black hole. There are several features in these backgrounds that are similar to the case of the plane AdS-Schwarzschild black hole. In particular, there is again a one parameter family of boundary conditions labeled by the self-adjoint parameter $z$ as before. However, now they lead to the same solution. The solution is a 'horizon state', i.e. it is

localized at the horizon. There seems to be no such non-trivial zero mode 
for the extremal Reissner-Nordstrom black hole.

Lastly, we would like to speculate on the possible interpretation of these irregular modes in the boundary theory. As first observed by [5], the modes with negative $k^{2}$ correspond to glueballs with mass $k^{2}$. This correspondence, when applied to the irregular states, seem to imply the existence of tachyonic

glueball states. Actually, such a scenario is not as exotic as it may appear to be at first sight. It was pointed out a long time ago by Savvidy [11], and also by Nielsen and collaborators [12 14 that the perturbative vacuum of $Q C D$ is unstable. Considering a translation invariant background for $\mathrm{SU}(2)$ gauge fields, they obtained the effective one-loop potential. This has the structure of a double well potential along with an imaginary term signalling the onset of instability. This persists in $\mathrm{SU}(\mathrm{N})$ theories and at finite temperature [24]. Our scenario resembles this phenomenon, which seems to be indicated by the appearance of these modes.

\section{Acknowledgements:}

We would like to thank A. P. Balachandran, S. Kalyana Rama, R. Parthasarathy, B. Sathiapalan and A. Sen for useful discussions. 
schzm.nb

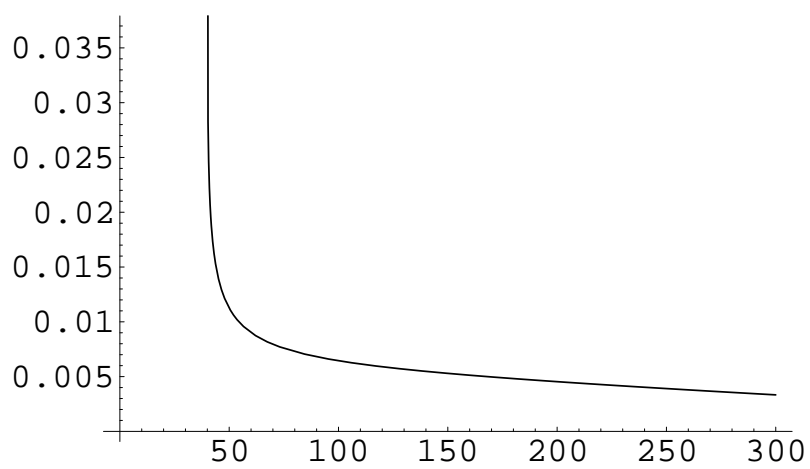

Figure 1.

Absolute value of zero mode solution for a Schwarzschild black hole with horizon radius $r_{+}=50$

ip.nb

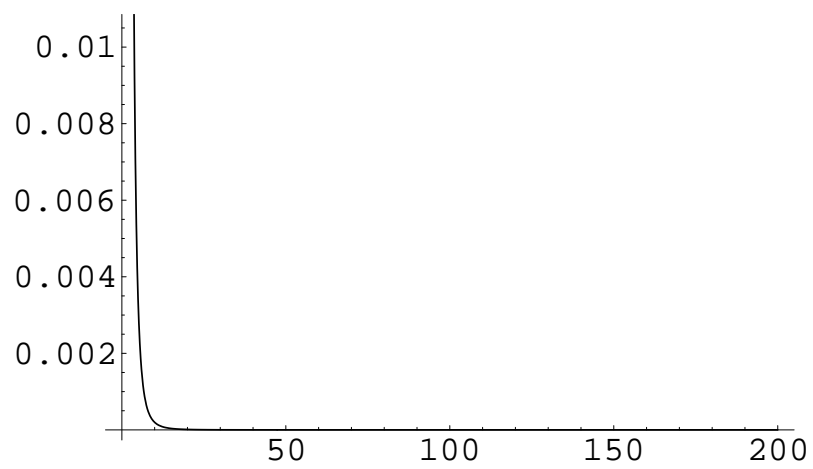

Figure 2. Absolute value of solution for $k^{2}=i$ as a function of $r$ 


\section{References}

[1] S. Chandrasekhar. The Mathematical Theory of Black Holes Clarendon Press, Oxford, 1983.

[2] R. Wald. J.Math.Phys 21:2802-2805, 1980.

[3] G.T. Horowitz, D. Marolf. Phys.Rev. D52:5670-5675, 1995. gr-qc/9504028.

[4] S. Hawking, D. Page. Commun.Math.Phys. 87:577, 1983.

[5] E. Witten. Adv.Theor.Math.Phys. 2:505-532, 1998. hep-th/9803131.

[6] C. Csáki, H. Ooguri, Y. Oz, J. Terning. JHEP 9901:017, 1999. hep-th/9806021.

[7] R. Koch, A. Jevicki, M. Mihailescu, J.P Nunes. Phys.Rev. D58:105009, 1998. hep-th/9806125.

[8] R.C. Brower, S.D. Mathur, C. Tan hep-th/9908196.

[9] S. Kalyana Rama, B. Sathiapalan. hep-th/9905219.

[10] G.T. Horowitz, V. Hubeny. hep-th/9909056.

[11] G. K. Savvidy. Phys.Lett. 71B:133-134, 1977.

[12] H. B. Nielsen and M. Ninomiya. Nucl.Phys. B156:1-28, 1979.

[13] N. K. Nielsen and P. Olesen. Nucl.Phys. B144:376-396, 1978.

[14] J. Ambjørn, N. K. Nielsen and P. Olesen. Nucl.Phys. B152:75-96, 1979.

[15] E. Witten. Phys.Rev. D44:314-324, 1991.

[16] M. Banados, C. Teitelboim, J. Zanelli, Phys.Rev.Lett 69:1849- ,1992.

[17] T. Jacobson. Phys.Rev. D57:4890-4898, 1998. hep-th/9705017.

[18] K.D. Kokkotas, B. Schmidt. Living Reviews in Relativity, Vol.2, 1999. www.livingreviews.org/Articles/Volume2/1999-2kokkotas

H. Nollert Class.Quant.Grav. 16:R159-R216, 1999.

[19] S. Chandrasekhar. Proc.Roy.Soc.London, Ser.A, 392:1-13, 1984. 
[20] M. Reed and B.Simon. Methods of Modern Mathematical Physics, volume I. Functional Analysis. Academic Press, New York, 1972.

[21] M. Reed and B.Simon. Methods of Modern Mathematical Physics, volume II. Fourier Analysis, Self-Adjointness. Academic Press, New York, 1975.

[22] K. Meetz. Nuovo Cimento 34:690, 1964.

[23] H. Narnhofer. Acta Physica Austriaca 40:306-322, 1974.

[24] D. Kay. Phys.Rev. D29:2409-2411, 1984. 